\title{
The Dombrock System: Linkage Relations with Other Blood Group Loci
}

\author{
PATRICIA TIPPETT, JUNE GAVIN, and RUTH SANGER \\ MRC Blood Group Unit, The Lister Institute, London SW1W 8RH
}

The recognition of the blood group system called Dombrock was reported in 1965 by Swanson and her colleagues. In 1967 Tippett gave an account of the genetics of the system which included an analysis of the linkage relations of the Do locus to other blood group loci, based on families tested in the MRC Blood Group Unit up to April 1966. The present account brings the linkage analysis up to the end of May 1972. As before, the calculations were by the method of Morton (1955) as instructed by Maynard Smith, Penrose, and Smith (1961).

The earlier series of families served to exclude close linkage between $D o$ and any of the other loci but in certain comparisons the lod scores were positive for recombination fractions ranging from $0 \cdot 2$ upwards, as may be seen in the Appendix and the Table. These positive scores were small but left open the possibility that a linkage might ultimately be detectable. The addition of the present series of families has reduced the possibility of linkage of Do with $R h$ and with Fy (Appendix) but increased the evidence for linkage between $D o$ and $M N S s$ (Table).

The antilogs of the total Do:MNSs lod scores in the Table, plotted in Fig. 1, show the relative probabilities of the recombination fractions when the scoring was through males, through females, and through either parent.

That the slight evidence of linkage between $D o$ and $M N S s$ is given when scored through doubly heterozygous females as well as males may be a warning that chance rather than linkage may be responsible for our results. The increased recombination rate in the human female, recognized by Cook (1964/1965) and by Renwick and Schulze (1964/1965), has now been observed in several linkages. If $\theta^{\prime}$ for the male were indeed about 0.33 as hinted by our data (Fig. 1) then the females would not be expected to give any indication of linkage. However, as Cook and his colleagues

Received 12 July 1972.

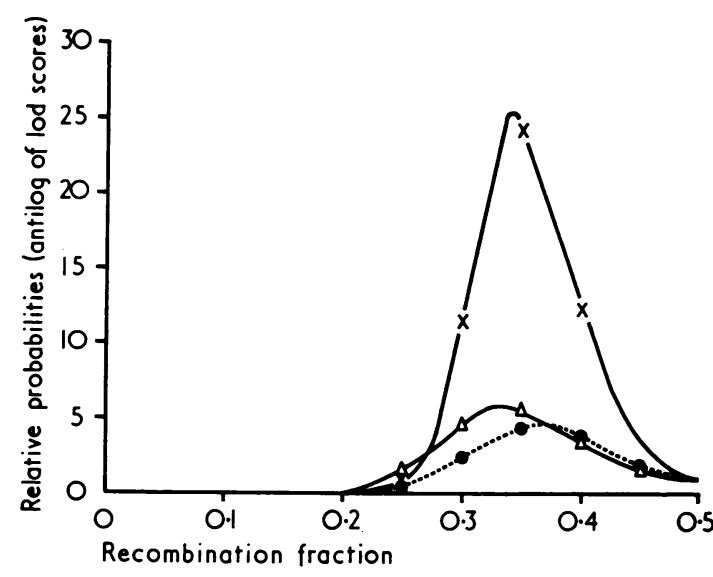

FIG. 1. Do and MNSs: relative probabilities of the various recombination fractions given in the Table. $\Delta=$ scored through males $\left(\theta^{\prime}=0.33\right) ; 0=$ scored through females $(\theta=0.37) ; x=$ scored through males and females $\left(\theta^{\prime}+\theta=0 \cdot 34\right)$.

(1971/1972) point out, an increased female rate of recombination does not apply to all of the linkages in the mouse (Dunn and Bennett, 1967) so may not apply to all in man.

The most straightforward evidence in favour of a Do:MNSs linkage is to be found in the Table in the phase-known non-recombinant and recombinant children of doubly heterozygous males (6 nonrecombinants: 2 recombinants) and doubly heterozygous females (9 non-recombinants: 3 recombinants). This ratio of 15:5 departs significantly from the 50:50 expected if there were no linkage $\left(\chi_{1}^{2}=5 \cdot 0, \mathrm{p}=0 \cdot 03\right)$.

If the testing of further families eventually confirms linkage between $D o$ and $M N S s$ then will be the time to try to organize the testing of families with the rare condition génodermatose scléro-atrophiante (sclerotylosis) the locus for which is known to be linked to MNSs (Mennecier, 1967; Deminatti et al, 1968). 
TABLE

A SUGGESTION OF LINKAGE BETWEEN Do AND MNSs: LOD SCORES

\begin{tabular}{|c|c|c|c|c|c|c|c|c|c|c|c|c|}
\hline \multirow{3}{*}{\begin{tabular}{l}
\multicolumn{1}{c}{$\begin{array}{c}\text { Doubly } \\
\text { Heterozygous } \\
\text { Parents }\end{array}$} \\
Males \\
First series \\
Present series \\
Sum \\
Antilogs
\end{tabular}} & \multirow{2}{*}{\multicolumn{2}{|c|}{$\begin{array}{l}\text { No. of Scoring } \\
\text { Parents: Children }\end{array}$}} & \multirow{2}{*}{\multicolumn{2}{|c|}{$\begin{array}{l}\text { Phase-known } \\
\text { Non-recombinant: } \\
\text { recombinant }\end{array}$}} & \multicolumn{8}{|c|}{ Recombination Fraction } \\
\hline & & & & & 0.05 & $0 \cdot 1$ & $0 \cdot 2$ & 0.25 & $0 \cdot 3$ & 0.35 & 0.4 & 0.45 \\
\hline & $\begin{array}{l}30 \\
17\end{array}$ & $\begin{array}{r}110 \\
48\end{array}$ & $\begin{array}{l}3 \\
3\end{array}$ & $\begin{array}{l}1 \\
1\end{array}$ & $\begin{array}{r}-11 \cdot 253 \\
-5 \cdot 242 \\
-16 \cdot 495\end{array}$ & $\begin{array}{l}-5 \cdot 014 \\
-2 \cdot 416 \\
-7 \cdot 430\end{array}$ & $\begin{array}{c}-0.559 \\
-0.396 \\
-0.955 \\
0.11\end{array}$ & $\begin{array}{l}+0.254 \\
-0.032 \\
+0.222 \\
1.67\end{array}$ & $\begin{array}{c}+0.540 \\
+0.130 \\
+0.670 \\
4.68\end{array}$ & $\begin{array}{l}+0.573 \\
+0.175 \\
+0.748 \\
\quad 5.60\end{array}$ & $\begin{array}{l}+0.382 \\
+0.146 \\
+0.528 \\
3.37\end{array}$ & $\begin{array}{l}+0.158 \\
+0.080 \\
+0.238 \\
1.73\end{array}$ \\
\hline $\begin{array}{l}\text { Females } \\
\text { First series } \\
\text { Present series } \\
\text { Sum } \\
\text { Antilogs }\end{array}$ & $\begin{array}{l}23 \\
16\end{array}$ & $\begin{array}{l}81 \\
51\end{array}$ & $\begin{array}{l}6 \\
3\end{array}$ & $\begin{array}{l}3 \\
0\end{array}$ & $\begin{array}{r}-13 \cdot 880 \\
-4 \cdot 790 \\
-18 \cdot 670\end{array}$ & $\begin{array}{l}-7 \cdot 458 \\
-1 \cdot 732 \\
-9 \cdot 190\end{array}$ & $\begin{array}{c}-2.256 \\
+0.370 \\
-1.886 \\
0.01\end{array}$ & $\begin{array}{c}-1.106 \\
+0.679 \\
-0.427 \\
0.37\end{array}$ & $\begin{array}{c}-0.348 \\
+0.734 \\
+0.386 \\
2.43\end{array}$ & $\begin{array}{c}+0.008 \\
+0.625 \\
+0.633 \\
4.30\end{array}$ & $\begin{array}{c}+0.148 \\
+0.416 \\
+0.564 \\
3.66\end{array}$ & $\begin{array}{l}+0.107 \\
+0.179 \\
+0.286 \\
1.93\end{array}$ \\
\hline $\begin{array}{l}\text { Males and } \\
\text { females } \\
\text { First series } \\
\text { Present series } \\
\text { Sum } \\
\text { Antilogs }\end{array}$ & $\begin{array}{l}53 \\
33\end{array}$ & $\begin{array}{r}191 \\
99\end{array}$ & $\begin{array}{l}9 \\
6\end{array}$ & $\begin{array}{l}4 \\
1\end{array}$ & $\begin{array}{l}-25 \cdot 133 \\
-10 \cdot 032 \\
-35 \cdot 165\end{array}$ & $\begin{array}{r}-12 \cdot 472 \\
-4 \cdot 148 \\
-16 \cdot 620\end{array}$ & $\begin{array}{c}-2.815 \\
-0.026 \\
-2.841 \\
0.00\end{array}$ & $\begin{array}{c}-0.852 \\
+0.647 \\
-0.205 \\
0.62\end{array}$ & $\begin{array}{l}+0.192 \\
+0.864 \\
+1.056 \\
11.38\end{array}$ & $\begin{array}{l}+0.581 \\
+0.800 \\
+1.381 \\
24.04\end{array}$ & $\begin{array}{c}+0.530 \\
+0.562 \\
+1.092 \\
12.36\end{array}$ & $\begin{array}{l}+0.265 \\
+0.259 \\
+0.524 \\
3.34\end{array}$ \\
\hline
\end{tabular}

We look back with regret on many fine families tested in the Unit in the last few years who were not tested for the Dombrock groups; but now having woken up to the possibility of linkage, we shall find time to use anti-Do ${ }^{\mathrm{a}}$ on all appropriate family samples.

\section{Summary}

A second series of families tested in the MRC Blood Group Unit for the Dombrock blood groups strengthens a hint of linkage between Do and $M N S$ s given by the first series: if the linkage should become established the present scores suggest that the most likely recombination fraction will prove to be about $0 \cdot 33$. Linkage between $D o$ and other blood group loci was either ruled out or made unlikely.

We thank Mrs Jane Swanson and Dr H. F. Polesky, of Minneapolis, who gave a generous supply of the original example of anti-Do ${ }^{a}$ serum from Mrs Dombrock, and very many physicians and cytogeneticists who for various reasons sent samples of blood from families. The anti$M$ and anti-N sera were kindly provided by the MRC Blood Group Reference Laboratory. We also thank the Sheffield and Glasgow blood transfusion centres for gifts of anti-human globulin sera particularly suited to the $\mathrm{Do}^{\mathrm{a}}$ : anti-Do ${ }^{\mathrm{a}}$ reaction.

\section{REFERENCES}

Cook, P. J. L. (1964/1965). The Lutheran-Secretor recombination fraction in man: a possible sex difference. Annals of Human Genetics, 28, 393-401.

Cook, P. J. L., Noades, J., Hopkinson, D. A., Robson, E. B., and Cleghorn, T. E. (1971/1972). Demonstration of a sex difference in recombination fraction in the loose linkage, $R h$ and $P G M_{1}$. Annals of Human Genetics, 35, 239-242.

Deminatti, M., Delmas-Marsalet, Y., Mennecier, M., Marquet, S., Agache, P., and Huriez, C. (1968). Etude du linkage probable entre une génodermatose a transmission autosomale dominante et le système de groupe sanguin MNSs. Annales de Génétique, 11, 217-224.

Dunn, L. C. and Bennett, D. (1967). Sex differences in recombination of linked genes in animals. Genetical Research, 9, 211220.

Maynard Smith, S., Penrose, L. S., and Smith, C. A. B. (1961). Mathematical Tables for Research Workers in Human Genetics, p. 74. Churchill, London.

Mennecier, M. (1967). Individualisation d'une Nouvelle Entité: la Génodermatose Scléro-Atrophiante et Kératodermique des Extrémités Fréquemment Dégénerative. Étude Clinique et Génétique (Possibilité de Linkage avec le Système MNSs). MD Thesis, p. 163. University of Lille.

Morton, N. E. (1955). Sequential tests for the detection of linkage American Fournal of Human Genetics, 7, 277-318.

Renwick, J. H. and Schulze, J. (1964/1965). Male and female recombination fractions for the nail-patella: $A B O$ linkage in man. Annals of Human Genetics, 28, 379-392.

Swanson, J., Polesky, H. F., Tippett, P., and Sanger, R. (1965). A 'new' blood group antigen, Do ${ }^{\mathrm{a}}$. Nature (London), 206, 313.

Tippett, P. (1967). Genetics of the Dombrock blood group system. fournal of Medical Genetics, 4, 7-11. 


\section{Appendix}

\section{Failure to Detect Linkage between the Dombrock Locus and Loci for Some Other Blood Group Systems: Lod Scores*}

\begin{tabular}{|c|c|c|c|c|c|c|c|c|c|}
\hline \multirow{4}{*}{$\begin{array}{l}\quad \text { Loci } \\
\\
A B O \\
\text { First series } \\
\text { Present series } \\
\text { Sum } \\
\end{array}$} & \multicolumn{9}{|c|}{ Transmission through Doubly Heterozygous Males } \\
\hline & \multirow{2}{*}{\multicolumn{2}{|c|}{$\begin{array}{l}\text { No. of Scoring } \\
\text { Fathers: Children }\end{array}$}} & \multirow{2}{*}{\multicolumn{2}{|c|}{$\begin{array}{l}\text { Phase-known } \\
\text { Non-recombinant: } \\
\text { Recombinant }\end{array}$}} & \multicolumn{5}{|c|}{ Recombination Fraction $\left(\theta^{\prime}\right)$} \\
\hline & & & & & 0.05 & 0.1 & 0.2 & 0.3 & 0.4 \\
\hline & $\begin{array}{l}28 \\
16\end{array}$ & 1111 & $\begin{array}{l}\mathbf{0} \\
\mathbf{0}\end{array}$ & $\begin{array}{l}5 \\
0\end{array}$ & $\begin{array}{l}-24 \cdot 250 \\
-10 \cdot 180 \\
-34 \cdot 430\end{array}$ & $\begin{array}{r}-14 \cdot 474 \\
-5 \cdot 790 \\
-20 \cdot 264 \\
\end{array}$ & $\begin{array}{l}-6 \cdot 079 \\
-2 \cdot 176 \\
-8 \cdot 255 \\
\end{array}$ & $\begin{array}{l}-2.454 \\
-0.732 \\
-3.186\end{array}$ & $\begin{array}{l}-0.722 \\
-0.151 \\
-0.873\end{array}$ \\
\hline $\begin{array}{l}P \\
\text { First series } \\
\text { Present series } \\
\text { Sum }\end{array}$ & $\begin{array}{l}5 \\
7\end{array}$ & $\begin{array}{l}19 \\
24\end{array}$ & $\begin{array}{l}\mathbf{0} \\
0\end{array}$ & $\begin{array}{l}\mathbf{0} \\
\mathbf{0}\end{array}$ & $\begin{array}{l}-3.694 \\
-0.170 \\
-3.864\end{array}$ & $\begin{array}{l}-2 \cdot 110 \\
+0.197 \\
-1.913\end{array}$ & $\begin{array}{l}-0.788 \\
+0.320 \\
-0.468\end{array}$ & $\begin{array}{l}-0.255 \\
+0.209 \\
-0.046\end{array}$ & $\begin{array}{l}-0.048 \\
+0.064 \\
+0.016\end{array}$ \\
\hline $\begin{array}{l}R h \\
\text { First series } \\
\text { Present series } \\
\text { Sum }\end{array}$ & $\begin{array}{l}27 \\
22\end{array}$ & $\begin{array}{r}100 \\
79\end{array}$ & $\begin{array}{l}4 \\
0\end{array}$ & $\begin{array}{l}\mathbf{0} \\
\mathbf{0}\end{array}$ & $\begin{array}{l}-13 \cdot 185 \\
-14 \cdot 367 \\
-27 \cdot 552\end{array}$ & $\begin{array}{r}-6 \cdot 404 \\
-7 \cdot 983 \\
-14 \cdot 387\end{array}$ & $\begin{array}{l}-1 \cdot 418 \\
-2 \cdot 806 \\
-4 \cdot 224\end{array}$ & $\begin{array}{l}+0.111 \\
-0.870 \\
-0.759\end{array}$ & $\begin{array}{l}+0.280 \\
-0.161 \\
+0.119\end{array}$ \\
\hline $\begin{array}{l}\text { Lu } \\
\text { First series } \\
\text { Present series } \\
\text { Sum }\end{array}$ & $\begin{array}{l}3 \\
4\end{array}$ & $\begin{array}{l}13 \\
11\end{array}$ & $\begin{array}{l}1 \\
0\end{array}$ & $\begin{array}{l}1 \\
0\end{array}$ & $\begin{array}{l}-3.396 \\
-1.522 \\
-4.918 \\
\end{array}$ & $\begin{array}{l}-2.042 \\
-0.948 \\
-2.990 \\
\end{array}$ & $\begin{array}{r}-0.858 \\
-0.423 \\
-1.276 \\
\end{array}$ & $\begin{array}{l}-0.345 \\
-0.167 \\
-0.512\end{array}$ & $\begin{array}{l}-0.074 \\
-0.039 \\
-0.113\end{array}$ \\
\hline $\begin{array}{l}\text { K } \\
\text { First series } \\
\text { Present series } \\
\text { Sum } \\
\end{array}$ & $\begin{array}{l}4 \\
3\end{array}$ & $\begin{array}{r}14 \\
8\end{array}$ & $\begin{array}{l}\mathbf{0} \\
\mathbf{0}\end{array}$ & $\begin{array}{l}\mathbf{0} \\
\mathbf{0}\end{array}$ & $\begin{array}{l}-2 \cdot 307 \\
-2 \cdot 884 \\
-5 \cdot 191 \\
\end{array}$ & $\begin{array}{l}-1 \cdot 302 \\
-1.775 \\
-3.077 \\
\end{array}$ & $\begin{array}{l}-0.488 \\
-0.776 \\
-1.264 \\
\end{array}$ & $\begin{array}{l}-0.167 \\
-0.303 \\
-0.470\end{array}$ & $\begin{array}{l}-0.035 \\
-0.071 \\
-0.106\end{array}$ \\
\hline $\begin{array}{l}\text { Fy } \\
\text { First series } \\
\text { Present series } \\
\text { Sum }\end{array}$ & $\begin{array}{r}12 \\
5\end{array}$ & $\begin{array}{l}36 \\
20\end{array}$ & $\begin{array}{l}0 \\
0\end{array}$ & $\begin{array}{l}\mathbf{0} \\
\mathbf{0}\end{array}$ & $\begin{array}{l}-1 \cdot 917 \\
-5 \cdot 512 \\
-7 \cdot 429 \\
\end{array}$ & $\begin{array}{l}-0.401 \\
-3.336 \\
-3.737 \\
\end{array}$ & $\begin{array}{l}+0.486 \\
-1.418 \\
-0.932 \\
\end{array}$ & $\begin{array}{l}+0.471 \\
-0.542 \\
-0.071 \\
\end{array}$ & $\begin{array}{l}+0.180 \\
-0.125 \\
+0.055\end{array}$ \\
\hline $\begin{array}{l}\text { fk } \\
\text { First series } \\
\text { Present series } \\
\text { Sum }\end{array}$ & $\begin{array}{r}13 \\
9\end{array}$ & $\begin{array}{l}40 \\
24\end{array}$ & $\begin{array}{l}1 \\
0\end{array}$ & $\begin{array}{l}2 \\
1\end{array}$ & $\begin{array}{r}-6.859 \\
-4.017 \\
-10.876 \\
\end{array}$ & $\begin{array}{l}-3.790 \\
-2.251 \\
-6.041 \\
\end{array}$ & $\begin{array}{r}-1.319 \\
-0.842 \\
-2.161 \\
\end{array}$ & $\begin{array}{r}-0.406 \\
-0.314 \\
-0.720 \\
\end{array}$ & $\begin{array}{l}-0.101 \\
-0.104 \\
-0.205 \\
\end{array}$ \\
\hline $\begin{array}{l}\text { Yt } \\
\text { First series } \\
\text { Present series } \\
\text { Sum }\end{array}$ & $\begin{array}{l}2 \\
0\end{array}$ & 8 & $\mathbf{0}$ & 0 & $\begin{array}{l}-2 \cdot 163 \\
-2 \cdot 163\end{array}$ & $\begin{array}{l}-1 \cdot 331 \\
-1 \cdot 331\end{array}$ & $\begin{array}{l}-0.582 \\
-0.582\end{array}$ & $\begin{array}{l}-0.227 \\
-0.227\end{array}$ & $\begin{array}{l}-0.053 \\
-0.053\end{array}$ \\
\hline $\begin{array}{l}\text { Co } \\
\text { First series } \\
\text { Present series } \\
\text { Sum } \\
\end{array}$ & $\begin{array}{l}0 \\
1\end{array}$ & 2 & 0 & 2 & $\begin{array}{l}-2 \cdot 000 \\
-2 \cdot 000 \\
\end{array}$ & $\begin{array}{r}-1 \cdot 398 \\
-1 \cdot 398 \\
\end{array}$ & $\begin{array}{l}-0.796 \\
-0.796 \\
\end{array}$ & $\begin{array}{l}-0.444 \\
-0.444 \\
\end{array}$ & $\begin{array}{l}-0.194 \\
-0.194\end{array}$ \\
\hline $\begin{array}{l}\text { Se } \\
\text { First series } \\
\text { Present series } \\
\text { Sum }\end{array}$ & $\begin{array}{l}2 \\
3\end{array}$ & $\begin{array}{l}6 \\
9\end{array}$ & 0 & 0 & $\begin{array}{l}-1 \cdot 145 \\
-1 \cdot 395 \\
-2 \cdot 540\end{array}$ & $\begin{array}{l}-0.635 \\
-0.849 \\
-1.484 \\
\end{array}$ & $\begin{array}{l}-0.226 \\
-0.364 \\
-0.590\end{array}$ & $\begin{array}{l}-0.072 \\
-0.140 \\
-0.212\end{array}$ & $\begin{array}{l}-0.015 \\
-0.033 \\
-0.048 \\
\end{array}$ \\
\hline $\begin{array}{l}\text { Le } \\
\text { First series } \\
\text { Present series } \\
\text { Sum }\end{array}$ & $\begin{array}{l}1 \\
0\end{array}$ & 3 & 0 & 0 & $\begin{array}{l}-0.769 \\
-0.769\end{array}$ & $\begin{array}{l}-0.482 \\
-0.482\end{array}$ & $\begin{array}{l}-0.216 \\
-0.216\end{array}$ & $\begin{array}{l}-0.086 \\
-0.086\end{array}$ & $\begin{array}{l}-0.020 \\
-0.020\end{array}$ \\
\hline
\end{tabular}

* In the present series, as in the first, practically all the information comes from $z_{1}$ scores. In the first series the comparatively uninformative $z_{2}$ and $z_{3}$ scores were also used on the relatively few families to which they applied: in the present series $z_{2}$ and $z_{3}$ scores were ignored, except when they could contribute to comparisons in which information was slight $(P, L u, K$ and $S e$ )

In this table the incidence of phase-known children is much higher when transmission is through females than when through males, and this requires explanation: very many of the families were primarily tested in connection with X-linkage and parents of mothers were specially sough out, thus providing 3 generation information; no special effort was made to get samples from parents of fathers. 


\begin{tabular}{|c|c|c|c|c|c|c|c|c|c|}
\hline \multicolumn{9}{|c|}{ Transmission through Doubly Heterozygous Females } & \multirow{3}{*}{ Loci } \\
\hline \multirow{2}{*}{\multicolumn{2}{|c|}{$\begin{array}{l}\text { No. of Scoring } \\
\text { Mothers: Children }\end{array}$}} & \multirow{2}{*}{\multicolumn{2}{|c|}{$\begin{array}{l}\text { Phase-known } \\
\text { Non-recombinant: } \\
\text { Recombinant }\end{array}$}} & \multicolumn{5}{|c|}{ Recombination Fraction $(\theta)$} & \\
\hline & & & & 0.05 & $0 \cdot 1$ & $0 \cdot 2$ & 0.3 & $0 \cdot 4$ & \\
\hline $\begin{array}{l}17 \\
14\end{array}$ & $\begin{array}{l}60 \\
47\end{array}$ & $\begin{array}{l}3 \\
3\end{array}$ & $\begin{array}{l}5 \\
1\end{array}$ & $\begin{array}{r}-12 \cdot 407 \\
-6.158 \\
-18.565\end{array}$ & $\begin{array}{r}-7 \cdot 167 \\
-2 \cdot 960 \\
-10 \cdot 127\end{array}$ & $\begin{array}{l}-2.735 \\
-0.544 \\
-3.279\end{array}$ & $\begin{array}{l}-0.952 \\
+0.160 \\
-0.792\end{array}$ & $\begin{array}{l}-0.228 \\
+0.182 \\
-0.046\end{array}$ & $\begin{array}{l}A B O \\
\text { First series } \\
\text { Present series } \\
\text { Sum }\end{array}$ \\
\hline $\begin{array}{l}3 \\
8\end{array}$ & $\begin{array}{l}20 \\
29\end{array}$ & $\begin{array}{l}0 \\
0\end{array}$ & $\begin{array}{l}0 \\
0\end{array}$ & $\begin{array}{l}-2 \cdot 012 \\
-4 \cdot 113 \\
-6 \cdot 125\end{array}$ & $\begin{array}{l}-1.006 \\
-2.444 \\
-3.450\end{array}$ & $\begin{array}{l}-0.230 \\
-0.979 \\
-1.209\end{array}$ & $\begin{array}{l}+0.009 \\
-0.377 \\
-0.368\end{array}$ & $\begin{array}{l}+0.027 \\
-0.070 \\
-0.043\end{array}$ & $\begin{array}{l}P \\
\text { First series } \\
\text { Present series } \\
\text { Sum }\end{array}$ \\
\hline $\begin{array}{l}21 \\
23\end{array}$ & $\begin{array}{l}72 \\
63\end{array}$ & $\begin{array}{l}5 \\
4\end{array}$ & $\begin{array}{l}3 \\
7\end{array}$ & $\begin{array}{r}-12.775 \\
-13.993 \\
-26.768 \\
\end{array}$ & $\begin{array}{r}-7 \cdot 079 \\
-8 \cdot 034 \\
-15 \cdot 113 \\
\end{array}$ & $\begin{array}{r}-2.429 \\
-3.117 \\
-5 \cdot 546 \\
\end{array}$ & $\begin{array}{l}-0.648 \\
-1.158 \\
-1.806 \\
\end{array}$ & $\begin{array}{l}-0.033 \\
-0.354 \\
-0.387 \\
\end{array}$ & $\begin{array}{l}\boldsymbol{R h} \\
\text { First series } \\
\text { Present series } \\
\text { Sum } \\
\end{array}$ \\
\hline$\frac{1}{2}$ & $\begin{array}{l}3 \\
5\end{array}$ & $\begin{array}{l}\mathbf{0} \\
\mathbf{0}\end{array}$ & $\begin{array}{l}0 \\
0\end{array}$ & $\begin{array}{r}-0.769 \\
+0.057 \\
-0.712 \\
\end{array}$ & $\begin{array}{l}-0.482 \\
+0.043 \\
-0.439\end{array}$ & $\begin{array}{l}-0.216 \\
+0.021 \\
-0.195\end{array}$ & $\begin{array}{l}-0.086 \\
+0.008 \\
-0.078\end{array}$ & $\begin{array}{r}-0.020 \\
+0.002 \\
-0.018\end{array}$ & $\begin{array}{l}\text { Lu } \\
\text { First series } \\
\text { Present series } \\
\text { Sum }\end{array}$ \\
\hline$\frac{1}{7}$ & $\begin{array}{r}7 \\
32\end{array}$ & $\begin{array}{l}0 \\
0\end{array}$ & $\begin{array}{l}0 \\
0\end{array}$ & $\begin{array}{l}-0.658 \\
-4.959 \\
-5.617\end{array}$ & $\begin{array}{l}-0 \cdot 392 \\
-2 \cdot 805 \\
-3 \cdot 197\end{array}$ & $\begin{array}{l}-0.162 \\
-0.996 \\
-1.158\end{array}$ & $\begin{array}{l}-0.061 \\
-0.281 \\
-0.342\end{array}$ & $\begin{array}{l}-0.013 \\
-0.039 \\
-0.051\end{array}$ & $\begin{array}{l}K \\
\text { First series } \\
\text { Present series } \\
\text { Sum }\end{array}$ \\
\hline $\begin{array}{r}14 \\
9\end{array}$ & $\begin{array}{l}49 \\
23\end{array}$ & $\begin{array}{l}2 \\
3\end{array}$ & $\begin{array}{l}4 \\
4\end{array}$ & $\begin{array}{r}-9.380 \\
-4.976 \\
-14.356 \\
\end{array}$ & $\begin{array}{l}-5 \cdot 275 \\
-2.946 \\
-8 \cdot 221 \\
\end{array}$ & $\begin{array}{r}-1.892 \\
-1.220 \\
-3.112 \\
\end{array}$ & $\begin{array}{l}-0.610 \\
-0.496 \\
-1.106 \\
\end{array}$ & $\begin{array}{l}-0.172 \\
-0.154 \\
-0.326 \\
\end{array}$ & $\begin{array}{l}\text { Fy } \\
\text { First series } \\
\text { Present series } \\
\text { Sum }\end{array}$ \\
\hline $\begin{array}{l}8 \\
6\end{array}$ & $\begin{array}{l}21 \\
17\end{array}$ & $\begin{array}{l}0 \\
2\end{array}$ & $\begin{array}{l}1 \\
2\end{array}$ & $\begin{array}{l}-4 \cdot 810 \\
-2 \cdot 557 \\
-7 \cdot 367 \\
\end{array}$ & $\begin{array}{l}-2.932 \\
-1.325 \\
-4.257 \\
\end{array}$ & $\begin{array}{l}-1.258 \\
-0.384 \\
-1.642\end{array}$ & $\begin{array}{l}-0.549 \\
-0.081 \\
-0.630 \\
\end{array}$ & $\begin{array}{l}-0.170 \\
-0.007 \\
-0.177 \\
\end{array}$ & $\begin{array}{l}\not{\not k} \\
\text { First series } \\
\text { Present series } \\
\text { Sum }\end{array}$ \\
\hline $\begin{array}{l}1 \\
0\end{array}$ & 8 & 0 & 0 & $\begin{array}{r}-1.906 \\
-1.906 \\
\end{array}$ & $\begin{array}{l}-1 \cdot 116 \\
-1 \cdot 116 \\
\end{array}$ & $\begin{array}{r}-0.448 \\
-0.448 \\
\end{array}$ & $\begin{array}{l}-0.163 \\
-0.163 \\
\end{array}$ & $\begin{array}{l}-0.036 \\
-0.036 \\
\end{array}$ & $\begin{array}{l}\text { Yt } \\
\text { First series } \\
\text { Present Series } \\
\text { Sum }\end{array}$ \\
\hline $\begin{array}{l}0 \\
2\end{array}$ & 6 & 1 & 1 & $\begin{array}{l}-2 \cdot 164 \\
-2 \cdot 164 \\
\end{array}$ & $\begin{array}{l}-1 \cdot 331 \\
-1 \cdot 331 \\
\end{array}$ & $\begin{array}{l}-0.582 \\
-0.582 \\
\end{array}$ & $\begin{array}{l}-0.227 \\
-0.227 \\
\end{array}$ & $\begin{array}{l}-0.053 \\
-0.053 \\
\end{array}$ & $\begin{array}{l}\text { Co } \\
\text { First Series } \\
\text { Present series } \\
\text { Sum }\end{array}$ \\
\hline $\begin{array}{l}3 \\
1\end{array}$ & $\begin{array}{r}11 \\
2\end{array}$ & $\begin{array}{l}0 \\
0\end{array}$ & $\begin{array}{l}0 \\
0\end{array}$ & $\begin{array}{l}-2.083 \\
-0.721 \\
-2.804 \\
\end{array}$ & $\begin{array}{l}-1.083 \\
-0.444 \\
-1.527\end{array}$ & $\begin{array}{l}-0.314 \\
-0.194 \\
-0.508\end{array}$ & $\begin{array}{l}-0.063 \\
-0.076 \\
-0.139 \\
\end{array}$ & $\begin{array}{r}-0.003 \\
-0.018 \\
-0.021\end{array}$ & $\begin{array}{l}\text { Se } \\
\text { First series } \\
\text { Present series } \\
\text { Sum } \\
\end{array}$ \\
\hline $\begin{array}{l}1 \\
0\end{array}$ & 3 & 0 & 0 & $\begin{array}{l}-0.769 \\
-0.769\end{array}$ & $\begin{array}{l}-0.482 \\
-0.482\end{array}$ & $\begin{array}{l}-0.216 \\
-0.216\end{array}$ & $\begin{array}{l}-0.086 \\
-0.086\end{array}$ & $\begin{array}{l}-0.020 \\
-0.020\end{array}$ & $\begin{array}{l}\text { Le } \\
\text { First series } \\
\text { Present series } \\
\text { Sum }\end{array}$ \\
\hline
\end{tabular}

